\title{
Certain Fractional Proportional Integral Inequalities via Convex Functions
}

\author{
Gauhar Rahman ${ }^{1}(\mathbb{D}$, Kottakkaran Sooppy Nisar $2, * \mathbb{C}$, Thabet Abdeljawad $3,4,5, * \mathbb{C}$ and \\ Samee Ullah ${ }^{6}$ \\ 1 Department of Mathematics, Shaheed Benazir Bhutto University, Sheringal, Upper Dir 18000, Pakistan; \\ gauhar55uom@gmail.com \\ 2 Department of Mathematics, College of Arts and Sciences, Prince Sattam bin Abdulaziz University, \\ Wadi Aldawaser 11991, Saudi Arabia \\ 3 Department of Mathematics and General Sciences, Prince Sultan University, Riyadh 12345, Saudi Arabia \\ Department of Medical Research, China Medical University, Taichung 40402, Taiwan \\ Department of Computer Science and Information Engineering, Asia University, Taichung 40402, Taiwan \\ 6 Department of Mathematics, University of Malakand, Lower Dir 18800, Chakdara, Pakistan; \\ sameeullah413@gmail.com \\ * Correspondence: n.sooppy@psau.edu.sa or ksnisar1@gmail.com (K.S.N.); tabdeljawad@psu.edu.sa (T.A.)
}

Received: 27 December 2019; Accepted: 6 February 2020; Published: 9 February 2020 updates

Abstract: The goal of this article is to establish some fractional proportional integral inequalities for convex functions by employing proportional fractional integral operators. In addition, we establish some classical integral inequalities as the special cases of our main findings.

Keywords: convex function; fractional integrals; proportional fractional integrals; inequalities; Qi inequality

MSC: 26A33; 26D10; 26D53; 05A30

\section{Introduction}

Integral inequalities play a vital role in the field of fractional differential equations. In the past few decades, researchers have paid their valuable consideration to this area. The significant developments in this area have been investigated, for example, [1-3], and [4] (cf. references cited therein). In [5], Ngo et al. established the following inequalities

$$
\int_{0}^{1} g^{\sigma+1}(t) d t \geq \int_{0}^{1} t^{\sigma} g(t) d t
$$

and

$$
\int_{0}^{1} g^{\sigma+1}(t) d t \geq \int_{0}^{1} t g^{\sigma}(t) d t
$$

where $\sigma>0$ and the positive continuous function $g$ on $[0,1]$ such that

$$
\int_{x}^{1} g(t) d t \geq \int_{x}^{1} t d t, x \in[0,1]
$$

Later on, Liu et al. [6] established the following inequalities

$$
\int_{a}^{b} g^{\sigma+\gamma}(t) d t \geq \int_{a}^{b}(t-a)^{\sigma} g^{\gamma}(t) d t,
$$


where $\sigma>0, \gamma>0$, and the positive continuous $g$ on $[a, b]$ is such that

$$
\int_{a}^{b} g^{\delta}(t) d t \geq \int_{a}^{b}(t-a)^{\delta} d t, \delta=\min (1, \gamma), t \in[a, b] .
$$

Liu et al. [7] derived two theorems for integral inequalities as follows:

Theorem 1. Suppose that the functions $f_{1}$ and $g_{1}$ are positive and continuous on $[a, b],(a<b)$ with $f_{1} \leq g_{1}$ on $[a, b]$ such that the function $\frac{f_{1}}{g_{1}},\left(g_{1} \neq 0\right)$ is decreasing and the function $f_{1}$ is increasing. Assume that the function $\Phi$ is a convex with $\Phi(0)=0$. Then, the following inequality holds

$$
\frac{\int_{a}^{b} f_{1}(t) d t}{\int_{a}^{b} g_{1}(t) d t} \geq \frac{\int_{a}^{b} \Phi\left(f_{1}(t)\right) d t}{\int_{a}^{b} \Phi\left(g_{1}(t)\right) d t} .
$$

Theorem 2. Suppose that the functions $f_{1}, f_{2}$, and $f_{3}$ be positive and continuous on $[a, b],(a<b)$ with $f_{1} \leq f_{2}$ on $[a, b]$ such that the function $\frac{f_{1}}{f_{2}},\left(f_{2} \neq 0\right)$ is decreasing and the functions $f_{1}$ and $f_{3}$ are increasing. Assume that the function $\Phi$ is a convex with $\Phi(0)=0$. Then, the following inequality holds

$$
\frac{\int_{a}^{b} f_{1}(t) d t}{\int_{a}^{b} f_{2}(t) d t} \geq \frac{\int_{a}^{b} \Phi\left(f_{1}(t)\right) f_{3}(t) d t}{\int_{a}^{b} \Phi\left(f_{2}(t)\right) f_{3}(t) d t} .
$$

The inequalities in Equations (1)-(3) and their various generalizations have gained attention of the researchers [8-12].

Furthermore, the research of fractional integral inequalities is also of prominent importance. In $[13,14]$, the authors presented some weighted Grüss type and new inequalities involving Riemann-Liouville (R-L) fractional integrals. In [15], Nisar et al. introduced many inequalities for extended gamma and confluent hypergeometric $k$-functions. Certain Gronwall inequalities for R-L and Hadamard $k$-fractional derivatives with applications are observed in [16]. The inequalities concerning the generalized $(k, \rho)$-fractional integral operators can be seen in [17].

The generalized fractional integral and Grüss type inequalities via generalized fractional integrals can be found in $[18,19]$. In [20], the authors examined the $(k, s)-\mathrm{R}-\mathrm{L}$ fractional integral and its applications. In [21], the authors presented generalized Hermite-Hadamard type inequalities through fractional integral operators. Dahmani [22] introduced some classes of fractional integral inequalities by employing a family of $n$ positive functions. Further the applications of fractional integral inequalities can be found $[23,24]$.

In the last few decades, the researchers have paid their valuable consideration to the field of fractional calculus. This field has received more attention from various researchers due to its wide applications in various fields. In the growth of fractional calculus, researchers concentrate to develop several fractional integral operators and their applications in distinct fields (see, e.g., [25-33]). Zaher et al. [34] presented a new fractional nonlocal model.

Such types of these new fractional integral operators promote the future study to develop certain new approaches to unify the fractional operators and secure fractional integral inequalities. Especially, several striking inequalities, properties, and applicability for the fractional conformable integrals and derivatives are recently studied by various researchers. We refer the interesting readers to the works by [35-44], and [45]. The applications of conformable derivative can be found in [46-49] (cf. references cited therein). 


\section{Preliminaries}

Jarad et al. [50] proposed the following left and right generalized proportional integral operators, which are sequentially defined by

$$
\left(a^{\xi} \mathcal{J}^{\xi, \delta} f\right)(\tau)=\frac{1}{\delta \xi \Gamma(\xi)} \int_{a}^{\tau} \exp \left[\frac{\delta-1}{\delta}(\tau-t)\right](\tau-t)^{\xi-1} f(t) d t, a<\tau
$$

and

$$
\left(\mathcal{J}_{b}^{\xi, \delta} f\right)(\tau)=\frac{1}{\delta \xi \Gamma(\xi)} \int_{\tau}^{b} \exp \left[\frac{\delta-1}{\delta}(t-\tau)\right](t-\tau)^{\xi-1} f(t) d t, \tau<b
$$

where the proportional index $\delta \in(0,1]$ and $\xi \in \mathbb{C}$ with $\operatorname{Re}(\xi)>0$ and $\Gamma(\tau)$ is the well-know gamma function defined by $\Gamma(\tau)=\int_{0}^{\infty} t^{\tau-1} e^{-t} d t[51-53]$.

Remark 1. Setting $\delta=1$ in Equations (4) and (5), we obtain the following left and right $R$-L:

$$
\left(a \mathcal{J}^{\xi} f\right)(\tau)=\frac{1}{\Gamma(\xi)} \int_{a}^{\tau}(\tau-t)^{\xi-1} f(t) d t, a<\tau
$$

and

$$
\left(\mathcal{J}_{b}^{\xi} f\right)(\tau)=\frac{1}{\Gamma(\xi)} \int_{\tau}^{b}(t-\tau)^{\xi-1} f(t) d t, \tau<b
$$

where $\xi \in \mathbb{C}$ with $\operatorname{Re}(\xi)>0$.

Recently, the generalized proportional derivative, and integral operators are established and studied in [54,55]. Certain new classes of integral inequalities for a class of $n(n \in \mathbb{N})$ positive continuous and decreasing functions on $[a, b]$ via generalized proportional fractional integrals can be found in the work of Rahman et al. [56]. The generalized Hadamard proportional fractional integrals and certain inequalities for convex functions by employing were recently proposed by Rahman et al. [57]. The bounds of proportional integrals in the sense of another function can be found in the work of Rahman et al. [58].

\section{Main Results}

In this section, we establish proportional fractional integral inequalities for convex functions by employing proportional fractional integral operators.

Theorem 3. Suppose that the functions $f$ and $g$ are positive and continuous on the interval $[a, b],(a<b)$ and $f \leq g$ on $[a, b]$. If the function $\frac{f}{g},(g \neq 0)$ is decreasing and the function $f$ is increasing on $[a, b]$, then, for any convex function $\Phi$ with $\Phi(0)=0$, the following inequality satisfies the proportional fractional integral operator given by Equation (4)

$$
\frac{{ }_{a} \mathcal{J}^{\xi, \delta}[f(\tau)]}{a \mathcal{J}^{\xi, \delta}[g(\tau)]} \geq \frac{\mathfrak{J}^{\xi, \delta}[\Phi(f(\tau))]}{a \mathcal{J}^{\xi, \delta}[\Phi(g(\tau))]}
$$

where $\delta \in(0,1], \xi \in \mathbb{C}$ with $\operatorname{Re}(\xi)>0$. 
Proof. Since $\Phi$ is convex function with $\Phi(0)=0$, the function $\frac{f(\tau)}{\tau}$ is increasing. As $f$ is increasing, the function $\frac{\Phi(f(\tau))}{f(\tau)}$ is also increasing. Obviously, $\frac{f(\tau)}{g(\tau)}$ is decreasing function. Thus, for all $\rho, \theta \in[a, b]$, we have

$$
\left(\frac{\Phi(f(\rho))}{f(\rho)}-\frac{\Phi(f(\theta))}{f(\theta)}\right)\left(\frac{f(\theta)}{g(\theta)}-\frac{f(\rho)}{g(\rho)}\right) \geq 0
$$

It follows that

$$
\frac{\Phi(f(\rho))}{f(\rho)} \frac{f(\theta)}{g(\theta)}+\frac{\Phi(f(\theta))}{f(\theta)} \frac{f(\rho)}{g(\rho)}-\frac{\Phi(f(\theta))}{f(\theta)} \frac{f(\theta)}{g(\theta)}-\frac{\Phi(f(\rho))}{f(\rho)} \frac{f(\rho)}{g(\rho)} \geq 0
$$

Multiplying Equation (7) by $g(\rho) g(\theta)$, we have

$$
\frac{\Phi(f(\rho))}{f(\rho)} f(\theta) g(\rho)+\frac{\Phi(f(\theta))}{f(\theta)} f(\rho) g(\theta)-\frac{\Phi(f(\theta))}{f(\theta)} f(\theta) g(\rho)-\frac{\Phi(f(\rho))}{f(\rho)} f(\rho) g(\theta) \geq 0 .
$$

Multiplying Equation (8) by $\frac{1}{\delta^{\tau} \Gamma(\xi)} \exp \left[\frac{\delta-1}{\delta}(\tau-\rho)\right](\tau-\rho)^{\xi-1}$, and integrating with respect to $\rho$ over $[a, \tau], a<\tau \leq b$, we have

$$
\begin{aligned}
& \frac{1}{\delta^{\xi} \Gamma(\xi)} \int_{a}^{\tau} \exp \left[\frac{\delta-1}{\delta}(\tau-\rho)\right](\tau-\rho)^{\xi-1} \frac{\Phi(f(\rho))}{f(\rho)} f(\theta) g(\rho) d \rho \\
+ & \frac{1}{\delta^{\xi} \Gamma(\xi)} \int_{a}^{\tau} \exp \left[\frac{\delta-1}{\delta}(\tau-\rho)\right](\tau-\rho)^{\xi-1} \frac{\Phi(f(\theta))}{f(\theta)} f(\rho) g(\theta) d \rho \\
- & \frac{1}{\delta^{\tau} \Gamma(\xi)} \int_{a}^{\tau} \exp \left[\frac{\delta-1}{\delta}(\tau-\rho)\right](\tau-\rho)^{\xi-1} \frac{\Phi(f(\theta))}{f(\theta)} f(\theta) g(\rho) d \rho \\
- & \frac{1}{\delta^{\xi} \Gamma(\xi)} \int_{a}^{\tau} \exp \left[\frac{\delta-1}{\delta}(\tau-\rho)\right](\tau-\rho)^{\xi-1} \frac{\Phi(f(\rho))}{f(\rho)} f(\rho) g(\theta) d \rho \geq 0 .
\end{aligned}
$$

Then, it follows that

$$
\begin{gathered}
f(\theta)_{a} \mathcal{J}^{\xi, \delta}\left(\frac{\Phi(f(\tau))}{f(\tau)} g(\tau)\right)+\left(\frac{\Phi(f(\theta))}{f(\theta)} g(\theta)\right)_{a} \mathcal{J}^{\xi, \delta}(f(\tau)) \\
-\left(\frac{\Phi(f(\theta))}{f(\theta)} f(\theta)\right)_{a} \mathcal{J}^{\xi, \delta}(g(\tau))-g(\theta)_{a} \mathcal{J}^{\xi, \delta}\left(\frac{\Phi(f(\tau))}{f(\tau)} f(\tau)\right) \geq 0 .
\end{gathered}
$$

Again, multiplying both sides of Equation (9) by $\frac{1}{\delta^{\tau} \Gamma(\xi)} \exp \left[\frac{\delta-1}{\delta}(\tau-\theta)\right](\tau-\theta)^{\xi-1}$, and integrating the resultant inequality with respect to $\theta$ over $[a, \tau], a<\tau \leq b$, we get

$$
\begin{aligned}
& { }_{a} \mathcal{J}^{\xi, \delta}(f(\tau))_{a} \mathcal{J}^{\xi, \delta}\left(\frac{\Phi(f(\tau))}{f(\tau)} g(\tau)\right)+{ }_{a} \mathcal{J}^{\xi, \delta}\left(\frac{\Phi(f(\tau))}{f(\tau)} g(\tau)\right){ }_{a} \mathcal{J}^{\xi, \delta}(f(\tau)) \\
\geq & a_{a} \mathcal{J}^{\xi, \delta}(g(\tau))_{a} \mathcal{J}^{\xi, \delta}(\Phi(f(\tau)))+{ }_{a} \mathcal{J}^{\xi, \delta}(\Phi(f(\tau)))_{a} \mathcal{J}^{\xi, \delta}(g(\tau)) .
\end{aligned}
$$

It follows that

$$
\frac{{ }_{a} \mathcal{J}^{\xi, \delta}(f(\tau))}{{ }_{a} \mathcal{J}^{\xi, \delta}(g(\tau))} \geq \frac{{ }_{a} \mathcal{J}^{\xi, \delta}(\Phi(f(\tau)))}{{ }_{a} \mathcal{J}^{\xi, \delta}\left(\frac{\Phi(f(\tau))}{f(\tau)} g(\tau)\right)}
$$

Now, since $f \leq g$ on $[a, b]$ and $\frac{\Phi(\tau)}{\tau}$ is an increasing function, for $\rho \in[a, \tau], a<\tau \leq b$, we have

$$
\frac{\Phi(f(\rho))}{f(\rho)} \leq \frac{\Phi(g(\rho))}{g(\rho)}
$$


Multiplying both sides of Equation (11) by $\frac{1}{\delta^{\tau} \Gamma(\xi)} \exp \left[\frac{\delta-1}{\delta}(\tau-\rho)\right](\tau-\rho)^{\xi-1} g(\rho)$ and integrating the resultant inequality with respect to $\rho$ over $[a, \tau], a<\tau \leq b$, we get

$$
\begin{aligned}
& \frac{1}{\delta^{\xi} \Gamma(\xi)} \int_{a}^{\tau} \exp \left[\frac{\delta-1}{\delta}(\tau-\rho)\right](\tau-\rho)^{\xi-1} \frac{\Phi(f(\rho))}{f(\rho)} g(\rho) d \rho \\
\leq & \frac{1}{\delta^{\xi} \Gamma(\xi)} \int_{a}^{\tau} \exp \left[\frac{\delta-1}{\delta}(\tau-\rho)\right](\tau-\rho)^{\xi-1} \frac{\Phi(g(\rho))}{g(\rho)} g(\rho) d \rho,
\end{aligned}
$$

which, in view of Equation (4), can be written as

$$
{ }_{a} \mathcal{J}^{\xi, \delta}\left(\frac{\Phi(f(\tau))}{f(\tau)} g(\tau)\right) \leq{ }_{a} \mathcal{J}^{\xi, \delta}(\Phi(g(\tau))) .
$$

Hence, from Equations (10) and (12), we get Equation (6).

Remark 2. Applying Theorem 3 for $\delta=1$, we get Theorem 3.1 proved by [59].

Remark 3. Applying Theorem 3 for $\xi=\delta=1$ and $x=b$, we get Theorem 1 .

Theorem 4. Suppose that the functions $f$ and $g$ are positive and continuous on $[a, b],(a<b)$ and $f \leq g$ on $[a, b]$. If the function $\frac{f}{g},(g \neq 0)$ is decreasing and the function $f$ is increasing on $[a, b]$, then, for any convex function $\Phi$ with $\Phi(0)=0$, the following inequality satisfies the proportional fractional integral operator given by Equation (4)

$$
\frac{{ }_{a} \mathcal{J}^{\xi, \delta}[f(\tau)]_{a} \mathcal{J}^{\lambda, \delta}[\Phi(g(\tau))]+{ }_{a} \mathcal{J}^{\lambda, \delta}[f(\tau)]_{a} \mathcal{J}^{\xi, \delta}[\Phi(g(\tau))]}{{ }_{a} \mathcal{J}^{\xi, \delta}[g(\tau)]_{a} \mathcal{J}^{\lambda, \delta}[\Phi(f(\tau))]+{ }_{a} \mathcal{J}^{\lambda, \delta}[g(\tau)]_{a} \mathcal{J}^{\xi, \delta}[\Phi(f(\tau))]} \geq 1,
$$

where $\delta \in(0,1], \xi, \lambda \in \mathbb{C}$ with $\operatorname{Re}(\xi)>0$ and $\operatorname{Re}(\lambda)>0$.

Proof. Since $\Phi$ is convex function with $\Phi(0)=0$, the function $\frac{f(\tau)}{\tau}$ is increasing. As $f$ is increasing, the function $\frac{\Phi(f(\tau))}{f(\tau)}$ is also increasing. Clearly, the function $\frac{f(\tau)}{g(\tau)}$ is decreasing for all $\rho, \theta \in[a, \tau], a<$ $\tau \leq b$. Multiplying Equation (9) by $\frac{1}{\delta^{\lambda} \Gamma(\lambda)} \exp \left[\frac{\delta-1}{\delta}(\tau-\theta)\right](\tau-\theta)^{\lambda-1}$ and integrating the resultant inequality with respect to $\theta$ over $[a, \tau], a<\tau \leq b$, we get

$$
\begin{aligned}
&{ }_{a} \mathcal{J}^{\lambda, \delta}(f(\tau))_{a} \mathcal{J}^{\xi, \delta}\left(\frac{\Phi(f(\tau))}{f(\tau)} g(\tau)\right)+{ }_{a} \mathcal{J}^{\lambda, \delta}\left(\frac{\Phi(f(\tau))}{f(\tau)} g(\tau)\right){ }_{a} \mathcal{J}^{\tilde{\xi}, \delta}(f(\tau)) \\
& \geq_{a} \mathcal{J}^{\xi, \delta}(g(\tau))_{a} \mathcal{J}^{\lambda, \delta}\left(\frac{\Phi(f(\tau)}{f(\tau)} f(\tau)\right)+{ }_{a} \mathcal{J}^{\xi, \delta}\left(\frac{\Phi(f(\tau)}{f(\tau)} f(\tau)\right){ }_{a} \mathcal{J}^{\lambda, \delta}(g(\tau)) .
\end{aligned}
$$

Now, since $f \leq g$ on $[a, b]$ and $\frac{\Phi(\tau)}{\tau}$ is an increasing function, for $\rho \in[a, \tau], a<\tau \leq b$, we have

$$
\frac{\Phi(f(\rho))}{f(\rho)} \leq \frac{\Phi(g(\rho))}{g(\rho)}
$$

Multiplying both sides of Equation (14) by $\frac{1}{\delta^{\tau} \Gamma(\xi)} \exp \left[\frac{\delta-1}{\delta}(\tau-\rho)\right](\tau-\rho)^{\tilde{\xi}-1} g(\rho)$ and integrating the resultant inequality with respect to $\rho$ over $[a, \tau], a<\tau \leq b$, we get

$$
\begin{aligned}
& \frac{1}{\delta^{\tau} \Gamma(\xi)} \int_{a}^{\tau} \exp \left[\frac{\delta-1}{\delta}(\tau-\rho)\right](\tau-\rho)^{\xi-1} \frac{\Phi(f(\rho))}{f(\rho)} g(\rho) d \rho \\
\leq & \frac{1}{\delta^{\xi} \Gamma(\xi)} \int_{a}^{\tau} \exp \left[\frac{\delta-1}{\delta}(\tau-\rho)\right](\tau-\rho)^{\xi-1} \frac{\Phi(g(\rho))}{g(\rho)} g(\rho) d \rho,
\end{aligned}
$$


which, in view of Equation (4), can be written as

$$
{ }_{a} \mathcal{J}^{\xi, \delta}\left(\frac{\Phi(f(\tau))}{f(\tau)} g(\tau)\right) \leq{ }_{a} \mathcal{J}^{\xi, \delta}(\Phi(g(\tau))) .
$$

Similarly, one can obtain

$$
{ }_{a} \mathcal{J}^{\lambda, \delta}\left(\frac{\Phi(f(\tau))}{f(\tau)} g(\tau)\right) \leq{ }_{a} \mathcal{J}^{\lambda, \delta}(\Phi(g(\tau))) .
$$

Hence, from Equations (12), (13), (15), and (16), we get the desired result.

Remark 4. Setting $\xi=\lambda$, Theorem 4 leads to Theorem 3.

Remark 5. Applying Theorem 4 for $\delta=1$, we get Theorem 3.3 proved by Dahmani [59].

Theorem 5. Suppose that the functions $f, h$, and $g$ are positive and continuous on $[a, b],(a<b)$ and $f \leq h$ on $[a, b]$. If the function $\frac{f}{g}$ is decreasing and the functions $f$ and $h$ are increasing on $[a, b]$, then, for any convex function $\Phi$ with $\Phi(0)=0$, the following inequality satisfies the proportional fractional integral operator given by Equation (4)

$$
\frac{{ }_{a} \mathcal{J}^{\xi, \delta}[f(\tau)]}{{ }_{a} \mathcal{J}^{\xi, \delta}[g(\tau)]} \geq \frac{{ }_{a} \mathcal{J}^{\xi, \delta}[\Phi(f(\tau)) h(\tau)]}{{ }_{a} \mathcal{J}^{\xi, \delta}[\Phi(g(\tau)) h(\tau)]}
$$

where $\delta \in(0,1], \xi \in \mathbb{C}$ with $\operatorname{Re}(\xi)>0$.

Proof. Since $\Phi$ is convex function such that $\Phi(0)=0$, the function $\frac{\Phi(\tau)}{\tau}$ is increasing. As the function $f$ is increasing, $\frac{\Phi(f(\tau))}{f(\tau)}$ is also increasing. Clearly, the function $\frac{f(\tau)}{g(\tau)}$ is decreasing for all $\rho, \theta \in[a, \tau], a<$ $\tau \leq b$.

$$
\left(\frac{\Phi(f(\rho))}{f(\rho)} h(\rho)-\frac{\Phi(f(\theta))}{f(\theta)} h(\theta)\right)(f(\theta) g(\rho)-f(\rho) g(\theta)) \geq 0 .
$$

It follows that

$$
\frac{\Phi(f(\rho)) h(\rho)}{f(\rho)} f(\theta) g(\rho)+\frac{\Phi(f(\theta)) h(\theta)}{f(\theta)} f(\rho) g(\theta)-\frac{\Phi(f(\theta)) h(\theta)}{f(\theta)} f(\theta) g(\rho)-\frac{\Phi(f(\rho)) h(\rho)}{f(\rho)} f(\rho) g(\theta) \geq 0 .
$$

Multiplying Equation (17) by $\frac{1}{\delta^{\tau} \Gamma(\xi)} \exp \left[\frac{\delta-1}{\delta}(\tau-\rho)\right](\tau-\rho)^{\xi-1}$ and integrating the resultant inequality with respect to $\rho$ over $[a, \tau], a<\tau \leq b$, we have

$$
\begin{aligned}
& \frac{1}{\delta^{\tau} \Gamma(\xi)} \int_{a}^{\tau} \exp \left[\frac{\delta-1}{\delta}(\tau-\rho)\right](\tau-\rho)^{\xi-1} \frac{\Phi(f(\rho))}{f(\rho)} f(\theta) g(\rho) h(\rho) d \rho \\
+ & \frac{1}{\delta^{\tau} \Gamma(\xi)} \int_{a}^{\tau} \exp \left[\frac{\delta-1}{\delta}(\tau-\rho)\right](\tau-\rho)^{\xi-1} \frac{\Phi(f(\theta))}{f(\theta)} f(\rho) g(\theta) h(\theta) d \rho \\
- & \frac{1}{\delta^{\tau} \Gamma(\xi)} \int_{a}^{\tau} \exp \left[\frac{\delta-1}{\delta}(\tau-\rho)\right](\tau-\rho)^{\xi-1} \frac{\Phi(f(\theta))}{f(\theta)} f(\theta) h(\theta) g(\rho) d \rho \\
- & \frac{1}{\delta^{\tau} \Gamma(\xi)} \int_{a}^{\tau} \exp \left[\frac{\delta-1}{\delta}(\tau-\rho)\right](\tau-\rho)^{\xi-1} \frac{\Phi(f(\rho))}{f(\rho)} f(\rho) h(\rho) g(\theta) d \rho \geq 0 .
\end{aligned}
$$


It follows that

$$
\begin{aligned}
& f(\theta)_{a} \mathcal{J}^{\xi, \delta}\left(\frac{\Phi(f(\tau))}{f(\tau)} g(\tau) h(\tau)\right)+\left(\frac{\Phi(f(\theta))}{f(\theta)} g(\theta) h(\theta)\right)_{a} \mathcal{J}^{\xi, \delta}(f(\tau)) \\
- & \left(\frac{\Phi(f(\theta))}{f(\theta)} f(\theta) h(\theta)\right){ }_{a} \mathcal{J}^{\xi, \delta}(g(\tau))-g(\theta)_{a} \mathcal{J}^{\xi, \delta}\left(\frac{\Phi(f(\tau))}{f(\tau)} f(\tau) h(\tau)\right) \geq 0 .
\end{aligned}
$$

Again, multiplying both sides of Equation (18) by $\frac{1}{\delta^{\tau} \Gamma(\xi)} \exp \left[\frac{\delta-1}{\delta}(\tau-\theta)\right](\tau-\theta)^{\xi-1}$ and integrating the resultant inequality with respect to $\theta$ over $[a, \tau], a<\tau \leq b$, we get

$$
\begin{aligned}
& { }_{a} \mathcal{J}^{\xi, \delta}(f(\tau))_{a} \mathcal{J}^{\xi, \delta}\left(\frac{\Phi(f(\tau))}{f(\tau)} g(\tau) h(\tau)\right)+{ }_{a} \mathcal{J}^{\xi, \delta}\left(\frac{\Phi(f(\tau))}{f(\tau)} g(\tau) h(\tau)\right){ }_{a} \mathcal{J}^{\xi, \delta}(f(\tau)) \\
\geq & { }_{a} \mathcal{J}^{\xi, \delta}(g(\tau))_{a} \mathcal{J}^{\xi, \delta}(\Phi(f(\tau)) h(\tau))+{ }_{a} \mathcal{J}^{\xi, \delta}(\Phi(f(\tau)) h(\tau))_{a} \mathcal{J}^{\xi, \delta}(g(\tau)) .
\end{aligned}
$$

It follows that

$$
\frac{{ }_{a} \mathcal{J}^{\xi, \delta}(f(\tau))}{{ }_{a} \mathcal{J}^{\xi, \delta}(g(\tau))} \geq \frac{{ }_{a} \mathcal{J}^{\xi, \delta}(\Phi(f(\tau)) h(\tau))}{{ }_{a} \mathcal{J}^{\xi, \delta}\left(\frac{\Phi(f(\tau))}{f(\tau)} g(\tau) h(\tau)\right)} .
$$

In addition, since $f \leq g$ on $[a, b]$ and $\frac{\Phi(\tau)}{\tau}$ is an increasing function, for $\eta, \theta \in[a, b]$, we have

$$
\frac{\Phi(f(\eta))}{f(\eta)} \leq \frac{\Phi(g(\eta))}{g(\eta)}
$$

Multiplying both sides of Equation (20) by $\frac{1}{\delta^{\xi} \Gamma(\xi)} \exp \left[\frac{\delta-1}{\delta}(\tau-\eta)\right](\tau-\eta)^{\xi-1} g(\eta) h(\eta)$ and integrating the resultant inequality with respect to $\eta$ over $[a, \tau], a<\tau \leq b$, we get

$$
\begin{aligned}
& \frac{1}{\delta^{\tau} \Gamma(\xi)} \int_{a}^{\tau} \exp \left[\frac{\delta-1}{\delta}(\tau-\eta)\right](\tau-\eta)^{\xi-1} \frac{\Phi(f(\eta))}{f(\eta)} g(\eta) h(\eta) d \eta \\
\leq & \frac{1}{\delta^{\tau} \Gamma(\xi)} \int_{a}^{\tau} \exp \left[\frac{\delta-1}{\delta}(\tau-\eta)\right](\tau-\eta)^{\xi-1} \frac{\Phi(g(\eta))}{g(\eta)} g(\eta) h(\eta) d \eta,
\end{aligned}
$$

which, in view of Equation (4), can be written as

$$
{ }_{a} \mathcal{J}^{\xi, \delta}\left(\frac{\Phi(f(\tau))}{f(\tau)} g(\tau) h(\tau)\right) \leq{ }_{a} \mathcal{J}^{\tilde{\zeta}, \delta}(\Phi(g(\tau)) h(\tau)) .
$$

Hence, from Equations (21) and (19), we obtain the required result.

Remark 6. Applying Theorem 5 for $\delta=1$, we get Theorem 3.5 proved by Dahmani [59].

Remark 7. Applying Theorem 5 for $\delta=\xi=1$ and $x=b$, we get Theorem 2 .

Theorem 6. Suppose that the functions $f, h$, and $g$ are positive and continuous on $[a, b],(a<b)$ and $f \leq g$ on $[a, b]$. If the function $\frac{f}{g}$ is decreasing and the functions $f$ and $h$ are increasing on $[a, b]$, then, for any convex function $\Phi$ with $\Phi(0)=0$, the following inequality satisfies the proportional fractional integral operator given by Equation (4)

$$
\frac{{ }_{a} \mathcal{J}^{\mathcal{\xi}, \delta}[f(\tau)]_{a} \mathcal{J}^{\lambda, \delta}[\Phi(g(\tau)) h(\tau)]+{ }_{a} \mathcal{J}^{\lambda, \delta}[f(\tau)]_{a} \mathcal{J}^{\xi, \delta}[\Phi(g(\tau)) h(\tau)]}{\mathcal{J}^{\xi, \delta}[g(\tau)]_{a} \mathcal{J}^{\lambda, \delta}[\Phi(f(\tau)) h(\tau)]+{ }_{a} \mathcal{J}^{\lambda, \delta}[g(\tau)]_{a} \mathcal{J}^{\xi, \delta}[\Phi(f(\tau)) h(\tau)]} \geq 1,
$$

where $\delta \in(0,1], \xi, \lambda \in \mathbb{C}$ with $\operatorname{Re}(\xi)>0$ and $\operatorname{Re}(\lambda)>0$. 
Proof. Multiplying both sides of Equation (18) by $\frac{1}{\delta^{\lambda} \Gamma(\lambda)} \exp \left[\frac{\delta-1}{\delta}(\tau-\theta)\right](\tau-\theta)^{\lambda-1}$ and integrating the resultant inequality with respect to $\theta$ over $[a, \tau], a<\tau \leq b$, we get

$$
\begin{aligned}
& { }_{a} \mathcal{J}^{\lambda, \delta}(f(\tau))_{a} \mathcal{J}^{\xi, \delta}\left(\frac{\Phi(f(\tau))}{f(\tau)} g(\tau) h(\tau)\right)+{ }_{a} \mathcal{J}^{\lambda, \delta}\left(\frac{\Phi(f(\tau))}{f(\tau)} g(\tau) h(\tau)\right){ }_{a} \mathcal{J}^{\xi, \delta}(f(\tau)) \\
\geq & a_{a} \mathcal{J}^{\xi, \delta}(g(\tau))_{a} \mathcal{J}^{\lambda, \delta}\left(\frac{\Phi(f(\tau))}{f(\tau)} f(\tau) h(\tau)\right)+{ }_{a} \mathcal{J}^{\xi, \delta}\left(\frac{\Phi(f(\tau))}{f(\tau)} f(\tau) h(\tau)\right){ }_{a} \mathcal{J}^{\lambda, \delta}(g(\tau)) .
\end{aligned}
$$

Since $f \leq g$ on $[a, b]$ and $\frac{\Phi(\tau)}{\tau}$ is an increasing function, for $\eta, \theta \in[1, x], a<\tau \leq b$, we have

$$
\frac{\Phi(f(\eta))}{f(\eta)} \leq \frac{\Phi(g(\eta))}{g(\eta)}
$$

Multiplying both sides of Equation (24) by $\frac{1}{\delta^{\tau} \Gamma(\xi)} \exp \left[\frac{\delta-1}{\delta}(\tau-\eta)\right](\tau-\eta)^{\xi-1} g(\eta) h(\eta), \eta \in$ $[a, x], a<\tau \leq b$ and integrating the resultant inequality with respect to $\eta$ over $[a, \tau], a<\tau \leq b$, we get

$$
\left.{ }_{a} \mathcal{J}^{\xi, \delta}\left(\frac{\Phi(f(\tau))}{f(\tau)} g(\tau) h(\tau)\right) \leq{ }_{a} \mathcal{J}^{\xi, \delta}(\Phi(g(\tau)) h(\tau))\right) .
$$

Similarly, one can obtain

$$
\left.{ }_{a} \mathcal{J}^{\eta, \delta}\left(\frac{\Phi(f(\tau))}{f(\tau)} g(\tau) h(\tau)\right) \leq{ }_{a} \mathcal{J}^{\eta, \delta}(\Phi(g(\tau)) h(\tau))\right) .
$$

Hence, from Equations (23), (25), and (26), we obtain the required inequality in Equation (22).

Remark 8. If we consider $\xi=\lambda$, then Theorem 6 leads to Theorem 5.

Remark 9. Applying Theorem 6 for $\delta=1$, we get Theorem 3.7 of Dahmani [59].

\section{Concluding Remarks}

Some interesting integral inequalities for convex functions were presented by Liu et al. ([7] Theorems 9 and 10). Later, Dahmani [59] improved these integral inequalities by utilizing the R-L fractional integral operator. Here, we present some new fractional proportional integral inequalities for convex functions by utilizing the proportional fractional integrals. In fact, we established the inequalities presented in Theorem 1 and Theorem 2 using the fractional proportional integrals, which are nonlocal and their orders depend on two indices: $\delta$, which is the proportional index, and $\xi$, which is the iterated index.

Author Contributions: All the authors contributed equally. All authors have read and agreed to the published version of the manuscript.

Funding: The third author would like to thank Prince Sultan University for the support through the research group "Nonlinear Analysis Methods in Applied Mathematics" (NAMAM), group number RG-DES-2017-01-17.

Conflicts of Interest: The authors declare that they have no competing interests.

\section{References}

1. Mitrinovic, D.S.; Pecaric, J.E.; Fink, A.M. Classical and New Inequalities in Analysis; Kluwer Academic Publishers: Dordrecht, The Netherlands, 1993.

2. Pachpatte, B.G. Mathematical Inequalities, 1st ed.; North-Holland Mathematical Library (Volume 67) (Book 67); Elsevier Science: Amsterdam, The Netherlands, 2005. 
3. Qi, F. Several integral inequalities. JIPAM 2000, 1, 19.

4. Sarikaya, M.Z.; Yildirim, H.; Saglam, A. On Hardy type integral inequality associated with the generalized translation. Int. J. Contemp. Math. Sci. 2006, 1, 333-340. [CrossRef]

5. Ngo, Q.A.; Thang, D.D.; Dat, T.T.; Tuan, D.A. Notes on an integral inequality. J. Inequal. Pure Appl. Math. 2006, 7, 120 .

6. Liu, W.J.; Cheng, G.S.; Li, C.C. Further development of an open problem concerning an integral inequality. JIPAM 2008, 9, 14.

7. Liu, W.J.; Ngǒ, Q.A.; Huy, V.N. Several interesting integral inequalities. J. Math. Inequal. 2009, 3, $201-212$. [CrossRef]

8. Bougoufa, L. An integral inequality similar to Qi inequality. JIPAM 2005, 6, 27.

9. Boukerrioua, K.; Guezane Lakoud, A. On an open question regarding an integral inequality. JIPAM $2007,8,77$.

10. Dahmani, Z.; Bedjaoui, N. Some generalized integral inequalities. J. Adv. Res. Appl. Math. 2011, 3, 58-66. [CrossRef]

11. Dahmani, Z.; Metakkel Elard, H. Generalizations of some integral inequalities using Riemann-Liouville operator. Int. J. Open Probl. Compt. Math. 2011, 4, 40-46.

12. Liu, W.J.; Li, C.C.; Dong, J.W. On an open problem concerning an integral inequality. JIPAM 2007, 8, 74.

13. Dahmani, Z.; Tabharit, L. On weighted Gruss type inequalities via fractional integration. J. Adv. Res. Pure Math. 2010, 2, 31-38. [CrossRef]

14. Dahmani, Z. New inequalities in fractional integrals. Int. J. Nonlinear Sci. 2010, 9, 493-497.

15. Nisar, K.S.; Qi, F.; Rahman, G.; Mubeen, S.; Arshad, M. Some inequalities involving the extended gamma function and the Kummer confluent hypergeometric k-function. J. Inequal. Appl. 2018, 2018, 135. [CrossRef] [PubMed]

16. Nisar, K.S.; Rahman, G.; Choi, J.; Mubeen, S.; Arshad, M. Certain Gronwall type inequalities associated with Riemann-Liouville k- and Hadamard k-fractional derivatives and their applications. East Asian Math. J. 2018, 34, 249-263.

17. Rahman, G.; Nisar, K.S.; Mubeen, S.; Choi, J. Certain Inequalities involving the $(k, \eta)$-fractional integral operator. Far East J. Math. Sci. (FJMS) 2018, 103, 1879-1888. [CrossRef]

18. Sarikaya, M.Z.; Dahmani, Z.; Kiris, M.E.; Ahmad, F. $(k, s)$-Riemann-Liouville fractional integral and applications. Hacet. J. Math. Stat. 2016, 45, 77-89. [CrossRef]

19. Set, E.; Tomar, M.; Sarikaya, M.Z. On generalized Grüss type inequalities for $k$-fractional integrals. Appl. Math. Comput. 2015, 269, 29-34. [CrossRef]

20. Sarikaya, M.Z.; Budak, H. Generalized Ostrowski type inequalities for local fractional integrals. Proc. Am. Math. Soc. 2017, 145, 1527-1538. [CrossRef]

21. Set, E.; Noor, M.A.; Awan, M.U.; Gözpinar, A. Generalized Hermite-Hadamard type inequalities involving fractional integral operators. J. Inequal. Appl. 2017, 169, 10. [CrossRef]

22. Dahmani, Z. New classes of integral inequalities of fractional order. LE MATEMATICHE 2014, LXIX, 237-247. [CrossRef]

23. Podlubny, I. Fractional Differential Equations; Academic Press: London, UK, 1999.

24. Samko, S.G.; Kilbas, A.A.; Marichev, O.I. Fractional Integrals and Derivatives, Theory and Applications; Taylor \& Francis: Abingdon, UK, 1993.

25. Abdeljawad, T. On Conformable Fractional Calculus. J. Comput. Appl. Math. 2015, 279, 57-66. [CrossRef]

26. Abdeljawad, T.; Baleanu, D. Monotonicity results for fractional difference operators with discrete exponential kernels. Adv. Differ. Equ. 2017, 2017, 78. [CrossRef]

27. Abdeljawad, T.; Baleanu, D. On Fractional Derivatives with Exponential Kernel and their Discrete Versions. Rep. Math. Phys. 2017, 80, 11-27. [CrossRef]

28. Anderson, D.R.; Ulness, D.J. Newly defined conformable derivatives. Adv. Dyn. Syst. Appl. 2015, 10, $109-137$.

29. Atangana, A.; Baleanu, D. New fractional derivatives with nonlocal and non-singular kernel. Theory and application to heat transfer model. Therm. Sci. 2016, 20, 763-769. [CrossRef]

30. Caputo, M.; Fabrizio, M. A new Definition of Fractional Derivative without Singular Kernel. Progr. Fract. Differ. Appl. 2015, 1, 73-85.

31. Jarad, F.; Ugurlu, E.; Abdeljawad, T.; Baleanu, D. On a new class of fractional operators. Adv. Differ. Equ. 2017, 2017, 247. [CrossRef] 
32. Khalil, R.; Horani, M.A.; Yousef, A.; Sababheh, M. A new definition of fractional derivative. J. Comput. Appl. Math. 2014, 264, 65-70. [CrossRef]

33. Losada, J.; Nieto, J.J. Properties of a New Fractional Derivative without Singular Kernel. Progr. Fract. Differ. Appl. 2015, 1, 87-92.

34. Rahimi, Z.; Sumelka, W.; Yang, X.J. A new fractional nonlocal model and its application in free vibration of Timoshenko and Euler-Bernoulli beams. Eur. Phys. J. Plus 2017, 132, 479. [CrossRef]

35. Khan, M.A.; Khurshid, Y.; Du, T.-S.; Chu, Y.-M. Generalization of Hermite-Hadamard type inequalities via conformable fractional integrals. J. Funct. Spaces 2018, 2018, 5357463.

36. Khan, M.A.; Iqbal, A.; Suleman, M.; Chu, Y.-M. Hermite-Hadamard type inequalities for fractional integrals via Green's function. J. Inequal. Appl. 2018, 2018, 161. [CrossRef] [PubMed]

37. Huang, C.J.; Rahman, G.; Nisar, K.S.; Ghaffar, A.; Qi, F. Some Inequalities of Hermite-Hadamard type for $k$-fractional conformable integrals. Aust. J. Math. Anal. Appl. 2019, 16, 1-9.

38. Khurshid, Y.; Khan, M.A.; Chu, Y.-M. Conformable integral inequalities of the Hermite-Hadamard type in terms of GG- and GA-2 convexities. J. Funct. Spaces 2019, 2019, 6926107. [CrossRef]

39. Khurshid, Y.; Khan, M.A.; Chu, Y.-M.; Khan, Z.A. Hermite-Hadamard-Fejer inequalities for conformable fractional integrals via preinvex functions. J. Funct. Spaces 2019, 2019, 3146210. [CrossRef]

40. Mubeen, S.; Habib, S.; Naeem, M.N. The Minkowski inequality involving generalized $k$-fractional conformable integral. J. Inequal. Appl. 2019, 2019, 81. [CrossRef]

41. Nisar, K.S.; Rahman, G.; Mehrez, K. Chebyshev type inequalities via generalized fractional conformable integrals. J. Inequal. Appl. 2019, 2019, 245. [CrossRef]

42. Niasr, K.S.; Tassadiq, A.; Rahman, G.; Khan, A. Some inequalities via fractional conformable integral operators. J. Inequal. Appl. 2019, 2019, 217. [CrossRef]

43. Qi, F.; Rahman, G.; Hussain, S.M.; Du, W.S.; Nisar, K.S. Some inequalities of Čebyšev type for conformable $k$-fractional integral operators. Symmetry 2018, 10, 614. [CrossRef]

44. Rahman, G.; Nisar, K.S.; Qi, F. Some new inequalities of the Gruss type for conformable fractional integrals. Aims Math. 2018, 3, 575-583. [CrossRef]

45. Rahman, G.; Ullah, Z.; Khan, A.; Set, E.; Nisar, K.S. Certain Chebyshev type inequalities involving fractional conformable integral operators. Mathematics 2019, 7, 364. [CrossRef]

46. Ortega, A.; Rosales, J.J. Newton's law of cooling with fractional conformable derivative. Revista Mexicana de Física 2018, 64, 172-175. [CrossRef]

47. Hammad, M.A.; Khalil, R. Abel's formula and Wronskian for conformable fractional differential equations. Int. J. Differ. Equ. Appl. 2014, 13, 177-183.

48. Ilie, M.; Biazar, J.; Ayati, Z. General solution of Bernoulli and Riccati fractional differential equations based on conformable fractional derivative. Int. J. Appl. Math. Res. 2017, 6, 49-51.

49. Meng, S.; Cui, Y. The extremal solution to conformable fractional differential equations involving integral boundary condition. Mathematics 2019, 7, 186; doi:10.3390/math7020186. [CrossRef]

50. Jarad, F.; Abdeljawad, T.; Alzabut, J. Generalized fractional derivatives generated by a class of local proportional derivatives. Eur. Phys. J. Spec. Top. 2017, 226, 3457-3471. [CrossRef]

51. Wang, M.-K.; Chu, H.-H.; Chu, Y.-M. Precise Bounds for the Weighted Hölder Mean of the Complete P-Elliptic Integrals. J. Math. Anal. Appl. 2019, 480. [CrossRef]

52. Yang, Z.-H.; Qian, W.-M.; Chu, Y.-M.; Zhang, W. On rational bounds for the gamma function. J. Inequal. Appl. 2017, 2017, 210. [CrossRef]

53. Yang, Z.-H.; Qian, W.-M.; Chu, Y.-M.; Zhang, W. Monotonicity rule for the quotient of two functions and its application. J. Inequal. Appl. 2017, 2017, 106. [CrossRef]

54. Alzabut, J.; Abdeljawad, T.; Jarad, F.; Sudsutad, W. A Gronwall inequality via the generalized proportional fractional derivative with applications. J. Inequal. Appl. 2019, 2019, 101. [CrossRef]

55. Rahman, G.; Khan, A.; Abdeljawad, T.; Nisar, K.S. The Minkowski inequalities via generalized proportional fractional integral operators. Adv. Differ. Equ. 2019, 2019, 287. [CrossRef]

56. Rahman, G.; Abdeljawad, T.; Khan, A.; Nisar, K.S. Some fractional proportional integral inequalities. J. Inequal. Appl. 2019, 2019, 244. [CrossRef]

57. Rahman, G.; Abdeljawad, T.; Jarad, F.; Khan, A.; Nisar, K.S. Certain inequalities via generalized proportional Hadamard fractional integral operators. Adv. Differ. Equ. 2019, 2019, 454. [CrossRef] 
58. Rahman, G.; Abdeljawad, T.; Jarad, F.; Nisar, K.S. Bounds of generalized proportional fractional integrals in general form via convex functions and their applications. Mathematics 2020, 8, 113. [CrossRef]

59. Dahmani, Z. A note on some new fractional results involving convex functions. Acta Math. Univ. Comen. 2012, LXXXI, 241-246.

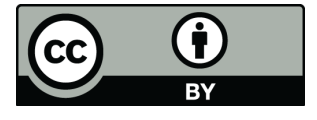

(C) 2020 by the authors. Licensee MDPI, Basel, Switzerland. This article is an open access article distributed under the terms and conditions of the Creative Commons Attribution (CC BY) license (http://creativecommons.org/licenses/by/4.0/). 